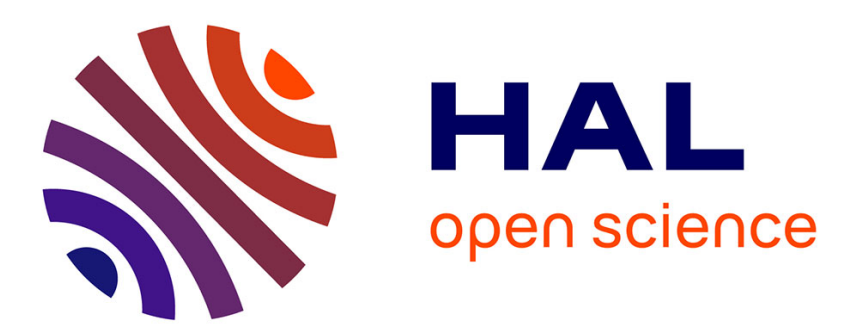

\title{
Lecteur de thermoluminescence permettant l'analyse des spectres d'émission
}

\author{
S. Sanzelle, Jean Fain, J.-C. Vennat
}

\section{To cite this version:}

S. Sanzelle, Jean Fain, J.-C. Vennat. Lecteur de thermoluminescence permettant l'analyse des spectres d'émission. Revue de Physique Appliquée, 1977, 12 (10), pp.1747-1751. 10.1051/rphysap:0197700120100174700 . jpa-00244397

\section{HAL Id: jpa-00244397 https://hal.science/jpa-00244397}

Submitted on 1 Jan 1977

HAL is a multi-disciplinary open access archive for the deposit and dissemination of scientific research documents, whether they are published or not. The documents may come from teaching and research institutions in France or abroad, or from public or private research centers.
L'archive ouverte pluridisciplinaire HAL, est destinée au dépôt et à la diffusion de documents scientifiques de niveau recherche, publiés ou non, émanant des établissements d'enseignement et de recherche français ou étrangers, des laboratoires publics ou privés. 
Classification

Physics Abstracts

$78.60 \mathrm{~K}-07.65$

\title{
LECTEUR DE THERMOLUMINESCENCE PERMETTANT L'ANALYSE DES SPECTRES D'ÉMISSION
}

\author{
S. SANZELLE, J. FAÏN et J.-C. VENNAT \\ Laboratoire de Physique Corpusculaire de l'Université de Clermont II, $\left(^{*}\right)$ \\ B.P. 45,63170 Aubière, France
}

(Reçu le 16 mai 1977, accepté le 28 juin 1977)

\begin{abstract}
Résumé. - Nous présentons un appareil conçu dans un but de recherche fondamentale pour obtenir des données quantitatives sur la thermoluminescence. Il peut être utilisé pour la mesure intégrale des émissions lumineuses et pour leur analyse en longueur d'onde. Nous indiquons les performances et le domaine d'emploi de l'appareil. Un exemple d'étude de spectre est donné.
\end{abstract}

Abstract. - This deals with a device designed for basic research to obtain quantitative data on thermoluminescence. It can be used for total measurement of light emissions and for wavelengths analysis. We give the performances and use range of this device. An example of spectrum analysis is given.

1. Introduction. - L'utilisation du phénomène de thermoluminescence comme moyen de dosimétrie s'est largement développée au cours des dix dernières années $[1,2]$. Dans le même temps, de nombreux lecteurs de thermoluminescence, dont certains sont commercialisés, ont été mis au point. Ils fonctionnent selon le principe suivant : le dosimètre est chauffé aussi linéairement que possible en fonction du temps, et la lumière émise est enregistrée par un photomultiplicateur placé près du dosimètre pour augmenter l'efficacité de détection. Ces appareils ont des domaines de fonctionnement bien définis : les matériaux utilisés comme dosimètres sont standardisés (géométrie, composition); l'émission lumineuse est enregistrée pour des températures inférieures à $200,250^{\circ} \mathrm{C}$; les doses d'utilisation des dosimètres sont faibles (inférieures à $100 \mathrm{krads}$ ); les irradiations étudiées sont généralement des $\gamma$ ou des $\beta$, donc des rayonnements de bas TEL (Transfert d'Énergie Linéique).

Dans ces conditions, le spectre en longueur d'onde de la lumière émise à un instant donné du chauffage peut être considéré comme connu et identique pour une catégorie de dosimètres. La mesure ne porte alors que sur le spectre en température (dit Glow Curve), et la dose est proportionnelle à l'intensité lumineuse totale (intégrée sur les températures).

Ces conditions de fonctionnement, valables pour des mesures dosimétriques de routine, sont trop

(*) Associé à l'IN2P3 (C.N.R.S.).

REVUE DE PHYSIQUE APPLIQUÉE. - T. 12, No 10, OCTOBRE 1977 restreintes lorsque la thermoluminescence est utilisée comme moyen d'investigation : par exemple. obtention d'informations sur les structures de dépôts d'énergie par différents types de particules [3], ou encore étude du phénomène de thermoluminescence lui-même [4]. On est alors exposé à sortir du domaine défini précédemment. En particulier, les spectres d'émission ne peuvent plus être considérés comme connus a priori. Or leur connaissance est intéressante pour la compréhension des phénomènes physiques et elle est indispensable pour une étude quantitative de ceux-ci. En effet, la réponse d'un photomultiplicateur est largement fonction de la longueur d'onde de la lumière incidente et la même énergie recueillie dans le rouge ou dans le violet donnera des signaux de sortie très différents.

L'appareil que nous présentons ici a été conçu dans un but de recherche, afin d'obtenir des données quantitatives dans un large domaine d'expérimentation.

2. Appareillage. - 2.1. CONCEPTION GÉNÉRALE. Étant données les conditions d'utilisation, le dispositif devra comporter :

- un porte-échantillon chauffant,

- un système dispersif permettant l'analyse spectrale de la lumière émise par l'échantillon à étudier : nous avons choisi un monochromateur à réseau. Il est à remarquer que dans les systèmes dispersifs optiques, l'image de l'objet est formée sur une fente d'entrée; l'intensité de la lumière transmise dépend beaucoup de la géométrie et du positionnement de 
l'échantillon, qui peuvent entraîner un recouvrement plus ou moins complet de l'image et de la fente,

- un photomultiplicateur de haute sensibilité et un enregistreur de données qui seront utilisés soit derrière le système dispersif (analyse spectrale) soit directement face à l'échantillon (mesure intégrale). Dans ce dernier cas, la mesure porte sur toute l'étendue spectrale du photomultiplicateur et est affranchie des incertitudes géométriques mentionnées précédemment.

Il est par ailleurs nécessaire de bien connaître les courbes de rendement en fonction de la longueur d'onde pour le photomultiplicateur et le monochromateur.

Les temps de chauffage sont courts : typiquement, on passe de la température ambiante à $300{ }^{\circ} \mathrm{C}$ en cinq minutes. Le spectre d'émission étant susceptible de changer avec la température, un balayage rapide et répété en longueur d'onde est nécessaire.

L'analyse d'une série d'échantillons ayant subi le même traitement comportera ainsi deux séquences : d'abord des lectures en mesure intégrale, ensuite des lectures en analyse spectrale qui préciseront la forme du spectre d'émission à chaque température. Un exemple d'application est présenté à la fin de ce texte.

2.2. ENCEINTE D'EXPÉRIMENTATION. - C'est un cube en aluminium, de $100 \mathrm{~mm}$ de côté, évidé de trois trous cylindriques forés perpendiculairement à chaque face. Les divers dispositifs sont fixés sur celles-ci avec interposition de joints toriques rendant l'ensemble étanche à la lumière et au vide. La figure 1

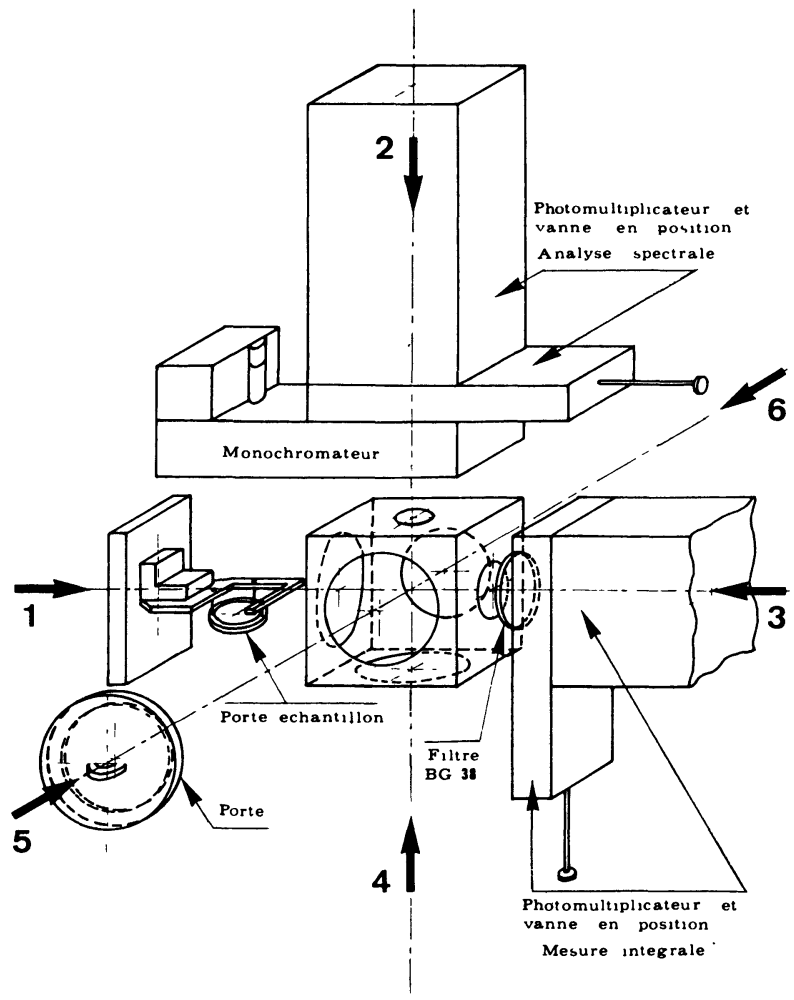

FIG. 1. - Plan schématique de l'appareil.

[Scheme of the apparatus.] donne une vue schématique de la disposition adoptée :

Le porte-échantillon est fixé en (1) par l'intermédiaire d'un flasque.

En (2) est montée une fenêtre de quartz suivie du monochromateur, d'une vanne optique et du photomultiplicateur (voie analyse spectrale)

Pour la voie mesure intégrale, en (3) est montée une autre fenêtre de quartz, derrière laquelle peuvent être fixés le photomultiplicateur et sa vanne optique.

L'ouverture (5) permet l'introduction rapide des échantillons.

Pour éviter les oxydations et améliorer la conduction thermique entre l'échantillon et son support, il est nécessaire d'effectuer les mesures en atmosphère d'azote. Aussi, en (4), un banc de pompage permet de faire le vide dans la chambre. L'azote gazeux est ensuite introduit jusqu'à obtenir la pression atmosphérique.

Enfin, l'ouverture (6) permet éventuellement le montage d'une source lumineuse de référence, ou celui d'un boîtier d'excitation en lumière ultraviolette pour effectuer des expériences de radiophotoluminescence àvec le même dispositif de détection.

2.3. Porte-ÉChantillon. - Conçu sur le principe de ceux réalisés par Keller et al. [5], il est constitué d'une résistance chauffante isolé Thermocoax (Type 1ZEZ Ac 10) de diamètre $1 \mathrm{~mm}$, enroulée en double spirale entre deux disques de cuivre et noyée dans de la brasure d'argent. L'ensemble se présente comme un disque de diamètre $40 \mathrm{~mm}$ et d'épaisseur $5 \mathrm{~mm}$ tenu en position horizontale par trois pointes d'acier inox, l'échantillon à analyser étant déposé au centre. La puissance maximale de $1 \mathrm{~kW}$, permet éventuellement une montée en température de $20^{\circ} \mathrm{C} / \mathrm{s}$.

Dans un logement ménagé sur la face inférieure est logé un couple thermo-électrique fer-constantan, qui délivre un signal proportionnel à la température.

2.4. ÉLECTRONIQUE DE PROGRAMMATION ET DE RÉGULATION DE TEMPÉRATURE. - Il a été employé un ensemble de contrôle ayant déjà donné de bons résultats [6]. Il comprend : un transmetteur qui traite l'information en provenance du thermo-couple; un régulateur à action proportionnelle, intégrale et différentielle; un programmateur, et un modulateur de puissance qui commande la résistance chauffante. Une grande souplesse d'emploi est ainsi obtenue; outre les montées linéaires en température, des paliers à température constante peuvent être facilement programmés. (Par exemple pour l'étude de la décroissance isotherme de la TL ou pour vider sélectivement avant lecture les pièges TL les moins profonds.)

2.5. Monochromateur. - Le monochromateur à réseau est du type SCHOEFFEL GM 100; il permet une analyse spectrale de 300 à $800 \mathrm{~nm}$. L'image de l'objet est formée à l'infini par une première lentille de quartz, puis sur la fente d'entrée par la première lentille du monochromateur. L'ouverture de ce 
dernier, égale à $\mathrm{f} / 4,7$, détermine celle de l'ensemble du dispositif optique. Trois jeux de fentes d'entrée peuvent être employés; leurs dimensions sont $0,5,1$ et $1,4 \mathrm{~mm}$ de largeur sur $6,5 \mathrm{~mm}$ de longueur; les résolutions correspondantes sont $4,5,9$ et $13 \mathrm{~nm}$.

Le réseau est entraîné par une vis micrométrique donnant une lecture directe et linéaire de la longueur d'onde. Pour obtenir un balayage automatique, la vis est entraînée par un moteur synchrone à deux sens de rotation; sur le même axe, un potentiomètre multi-tours, convenablement alimenté par une source de tension permet la conversion du nombre de tours en différence de potentiel. Cette dernière est ensuite envoyée sur la voie $\mathrm{X}$ d'une table traçante. Des interrupteurs fin de course dont la position est réglable limitent le domaine de longueur d'onde à analyser et inversent le sens de rotation du moteur. Les vitesses de balayage utilisées correspondent à 12,5 et $50 \mathrm{~nm} / \mathrm{s}$.

2.6. DÉTECTION ET ENREGISTREMENT. - Étant données les faibles intensités lumineuses en jeu, il fallait choisir un photomultiplicateur de rendement quantique élevé et de faible courant d'obscurité. Il est de type RCA C $31000 \mathrm{~A}$ à photocathode multialkali ayant une étendue spectrale de 260 à $800 \mathrm{~nm}$, avec un rendement quantique typique de $24 \%$ à $400 \mathrm{~nm}$. Placé dans une enceinte fermée par une vanne optique, il peut être refroidi à $-15^{\circ} \mathrm{C}$ par des éléments à effet Peltier. L'expérience a montré que les meilleures conditions de fonctionnement étaient obtenues pour des valeurs plutôt basses de l'alimentation haute tension $(1100 \mathrm{~V})$.

Sur la figure 1 sont indiquées les deux positions possibles. La position horizontale correspond à la lecture en mesure intégrale. La lumière émise par l'échantillon lors de son chauffage est alors réfléchie à $90^{\circ}$ vers la fenêtre d'entrée du photomultiplicateur par l'intermédiaire d'un miroir aluminisé amovible, placé au-dessus du porte-échantillon. Sur le trajet lumineux est interposé un filtre BG 38 LEITZ de $3 \mathrm{~mm}$ d'épaisseur pour diminuer le rayonnement thermique atteignant la photocathode.

La position verticale est utilisée en analyse spectrale. La lentille de focalisation envoie un faisceau de lumière parallèle sur le monochromateur, au-dessus duquel est monté le photomultiplicateur.

Dans les deux positions, le courant d'anode est mesuré par un pico-ampèremètre, comportant une sortie analogique, qui est reliée à la voie $\mathrm{Y}$ d'une table traçante. Pour obtenir les spectres d'intensité lumineuse en fonction de la température (Glow curves), en mesure intégrale, il suffit d'envoyer le signal du thermo-couple sur la voie $\mathbf{X}$ de cette table.

Enfin, un dispositif de sécurité interdit l'ouverture intempestive de l'enceinte lorsque la vanne optique du photomultiplicateur n'est pas fermée, et vice versa.

3. Étalonnage. - Comme il a déjà été indiqué, un étalonnage du dispositif est indispensable. Les courbes de rendement en fonction de la longueur d'onde données par les constructeurs pour le monochromateur ou le photomultiplicateur ne sont que des données typiques, relatives à un ensemble de matériel produit, et n'ont qu'une valeur indicative.

Pour établir ces courbes d'étalonnage, une source de lumière étalon est nécessaire. Il a été utilisé ici une lampe à incandescence spéciale (comportant un ruban de tungstène poli comme filament) alimentée sous tension stabilisée et dont la température de couleur était $2392 \pm 10 \mathrm{~K}$. Le spectre en était donc connu.

$\mathrm{La}$ réponse fournie par la voie analyse spectrale a permis la déduction de la courbe d'étalonnage correspondante. Ensuite un deuxième monochromateur a été employé devant la source pour limiter un étroit domaine de longueur d'onde; la caractéristique de transmission du monochromateur lié à la voie analyse spectrale est obtenue en faisant le rapport des intensités lumineuses reçues sur les deux voies.

On déduit ensuite de ces résultats la réponse spectrale du photomultiplicateur. Les courbes de réponse des deux voies sont ainsi obtenues en valeur relative, avec une précision estimée à $\pm 3 \%$.

Le photomultiplicateur est ensuite étalonné en valeur absolue toujours à partir de la même source étalon, grâce à un dispositif optique adéquat, pour une longueur d'onde de $5330 \AA$, permettant ainsi d'établir sa courbe de sensibilité absolue, en ampère/ watt, dans les conditions d'utilisation correspondantes (température ambiante, alimentation sous $1100 \mathrm{~V}$ ).

Enfin, la stabilité du photomultiplicateur est contrôlée fréquemment à l'aide de petites sources lumineuses de références constituées d'une source radioactive et d'un scintillateur, qui peuvent être placées sur le porte-échantillon.

4. Performances. - La géométrie adoptée pour notre dispositif (photomultiplicateur relativement éloigné de l'échantillon) n'est pas adaptée à la mesure des très faibles doses, domaine qui est plutôt du ressort de la dosimétrie. Néanmoins, de bons résultats sont encore obtenus en mesure intégrale à $5 \mathrm{rad}$. avec les dosimètres TLD-100 Harshaw ( $\mathrm{LiF}: \mathrm{Mg}: \mathrm{Ti}$ ) et ce pour un taux de chauffe de $1,2^{\circ} \mathrm{C} / \mathrm{s}$.

Toutes les vitesses de chauffe inférieures à $1{ }^{\circ} \mathrm{C} / \mathrm{s}$ peuvent être programmées. La montée en température est linéaire à $\pm 1 \%$ près et reproductible à mieux de $1 \%$. Les paliers de températures sont affichés avec une incertitude de $\pm 1^{\circ} \mathrm{C}$, et leur stabilité est du même ordre. La figure 2 montre quelques traitements thermiques typiques.

Le retard thermique entre le thermo-couple et l'échantillon est inférieur à $1 \mathrm{~s}$ si la géométrie de ce dernier est bonne (cristal à faces bien planes). La température des pics de TL est ainsi connue à $\pm 2^{\circ} \mathrm{C}$ près dans le cas d'un taux de chauffe égal à $1,2^{\circ} \mathrm{C} / \mathrm{s}$. Une valeur de $196{ }^{\circ} \mathrm{C}$ est ainsi obtenue pour le pic 


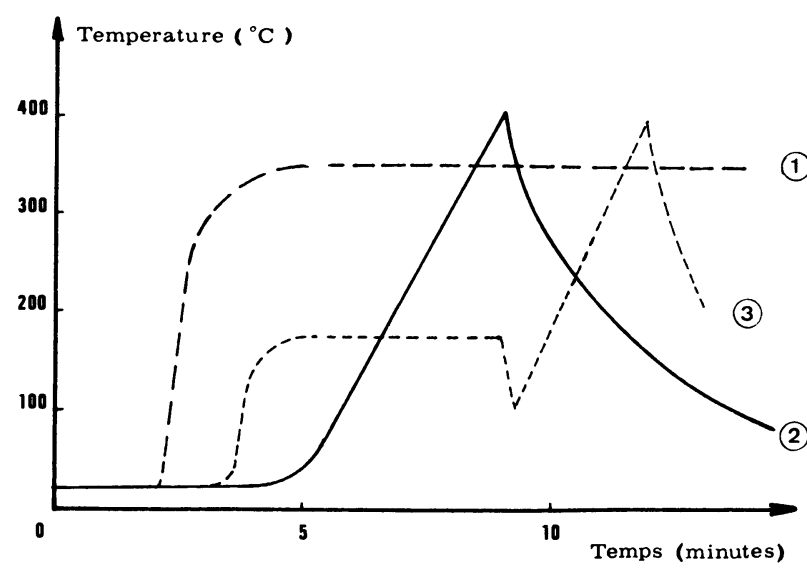

FIG. 2. - Traitements thermiques typiques : 1. palier à $350^{\circ} \mathrm{C}$ pour l'étude de la décroissance isotherme, 2. montée linéaire simple, suivie du refroidissement, 3. montée linéaire précédée d'un palier à $175^{\circ} \mathrm{C}$ pour vider les pièges TL les moins profonds.

[Typical heat treatment : 1 . level at $350^{\circ} \mathrm{C}$ for studying the isothermal decrease, 2. simple linear rise, before cooling, 3. linear rise after a level at $175^{\circ} \mathrm{C}$ to empty the shallowest TL traps.]

dominant du LiF TLD-100, et $270^{\circ} \mathrm{C}$ pour le $\mathrm{CaF}_{2}: \mathrm{Mn}$ (TLD 400 Harshaw).

La reproductibilité obtenue avec la partie optique seule de l'appareillage (mesures portant sur des sources lumineuses non thermoluminescentes) est de l'ordre de $1 \%$. Une série de mesures effectuées sur des dosimètres $\mathrm{TL} \mathrm{CaF}_{2}: \mathrm{Mn}$ ayant reçu la même dose (950 rads déposés par des $\gamma$ du $\left.{ }^{60} \mathrm{Co}\right)$ a donné une dispersion de $4 \%$ (écart quadratique moyen). Une partie de celle-ci est probablement due à l'appareillage : variation de réflectivité du porte-échantillon, inégalités de chauffage dues à des mauvais contacts thermiques, etc. mais le rôle de la dispersion en sensibilité des échantillons eux-mêmes n'est certainement pas négligeable.

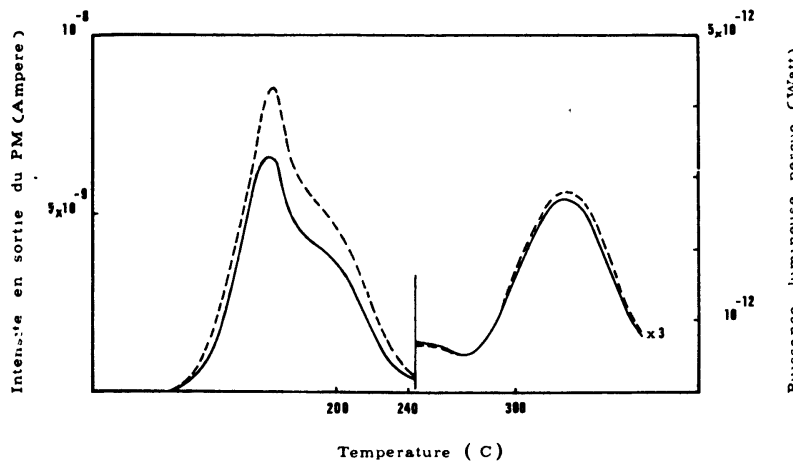

Fig. 3. - Intensité lumineuse émise en fonction de la température par un cristal de $\mathrm{CaF}_{2}$ : Dy irradié aux du ${ }^{60} \mathrm{Co}$ avec un taux de chauffe de $1,1^{\circ} \mathrm{C} / \mathrm{s}$, - données en ampères obtenues directement en sortie du photo-multiplicateur, ------ courbe corrigée, en watts.

[Light intensity vs temperature : the light is emitted by an irradiated $\left(\Upsilon\right.$ of $\left.{ }^{60} \mathrm{Co}\right)$ Crystal of $\mathrm{CaF}_{2}:$ Dy, heating rate $1.1^{\circ} \mathrm{C} . \mathrm{s}^{-1}$, - data in ampere directly received at the exit of the PM, -.-.-- corrected curve, in watt.]
5. Exemple d'analyse spectrale. - Sur la figure 3 est tracée la courbe donnant le signal en fonction de la température (Glow-curve) pour le $\mathrm{CaF}_{2}$ : Dy (dosimètre Harshaw TLD-200) telle qu'elle est obtenue à la sortie de l'appareillage en mesure intégrale avec un taux de chauffe de $1,2^{\circ} \mathrm{C} / \mathrm{s}$.

L'analyse spectrale d'un échantillon identique dans les mêmes conditions montre qu'il y a deux domaines de spectres d'émission : à moins de $240^{\circ} \mathrm{C}$ on observe (Fig. 4) les raies correspondant aux transitions du Dy ${ }^{3+}$; au-dessus (Fig. 5) il n'y a qu'un maximum unique, indiquant qu'il y a eu un changement de processus physique. Ces courbes sont ensuite corrigées pour tenir compte de l'efficacité en fonction de la

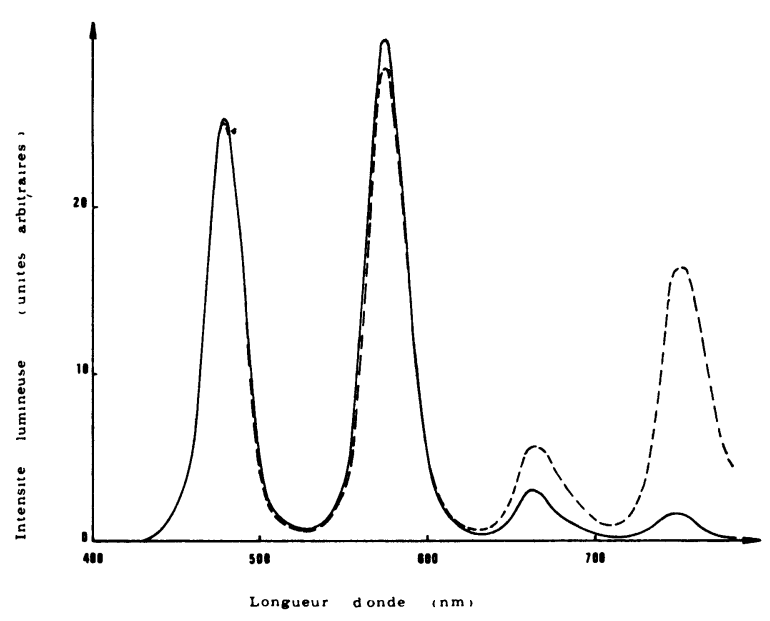

Fig. 4. - Spectre d'émission du $\mathrm{CaF}_{2}$ : Dy pour le domaine de température au-dessous de $240^{\circ} \mathrm{C}:-$ courbe brute, ----- courbe corrigée.

[Emissive spectrum from $\mathrm{CaF}_{2}: \mathrm{Dy}$ for temperature range below $240^{\circ} \mathrm{C}:-$ experimental curve, ------ corrected curve.]

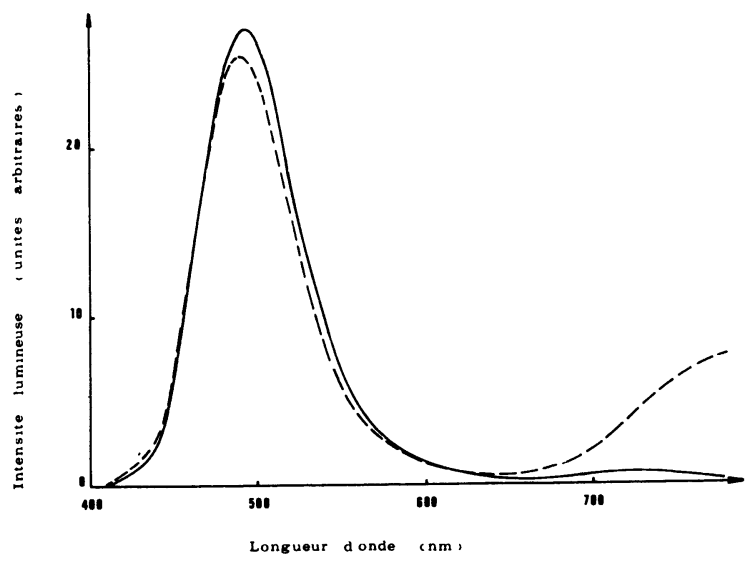

Fig. 5. - Spectre d'émission du $\mathrm{CaF}_{2}$ : Dy dans le domaine de température au-dessus de $240{ }^{\circ} \mathrm{C}:-$ courbe brute, ------ courbe corrigée.

[Emissive spectrum from $\mathrm{CaF}_{2}:$ Dy for temperature higher than $240^{\circ} \mathrm{C}$ : — - experimental curve, ----- corrected curve.] 
longueur d'onde sur la voie analyse spectrale et obtenir la véritable forme des spectres (pointillés). La connaissance de ces derniers et de la réponse absolue en fonction de la longueur d'onde sur la voie mesure intégrale permet de calculer la sensibilité sur celle-ci pour chacun des deux domaines : elle est dans les conditions d'utilisation choisies ici de $1530 \mathrm{~A} / \mathrm{W}$ dans le premier domaine et de $1900 \mathrm{~A} / \mathrm{W}$ dans le deuxième. La Glow curve peut alors être retracée sur la figure 3 en termes de puissance lumineuse absolue atteignant le photomultiplicateur et non plus d'intensité en sortie de celui-ci. La connaissance des caractéristiques géométriques de l'appareillage permet ensuite d'évaluer l'énergie totale libérée par l'échantillon sous forme de lumière dans le domaine de longueur d'onde analysé.

\section{Bibliographie}

[1] Proc. of the Third Int. Conf. on Luminescence Dosimetry, held at the Danish AEC Research, Establishment Riso 1114 October 1971

[2] Proc. of the Fourth Int. Conf. on Luminescence Dosimetry, Krakow, Poland, 27-31 August 1974

[3] Zimmerman D. W., Radiat. Eff. 14 (1970) 81.
[4] Fain J., Monnin M., International Workshop on Thermally Stimulated Processes, Montpellier (1976), à paraître dans .Journal of Electrostatics.

[5] Keller P., Lapraz D. et Iaconni P., Mod. Geol., 2 (1971) 71.

[6] Sanzelle S., Vennat J. C. et Gulllemain C., Revue Phys. Appl. 10 (1975) 471. 